\title{
Robust constrained MPG stabilization of a CSTR
}

\author{
Juraj Oravec, Monika Bakošová \\ Slovak University of Technology in Bratislava, Faculty of Chemical and Food Technology, \\ Institute of Information Engineering, Automation, and Mathematics, \\ Radlinského 9, 81237 Bratislava, Slovak Republic \\ juraj.oravec@stuba.sk
}

\begin{abstract}
The paper addresses a case study of robust stabilization of a continuous stirred tank reactor using robust model-based predictive control with constrained input variables. One exothermic reaction runs in the reaction mixture and the reactor is modelled in the form of an uncertain polytopic system. The control approach is based on solution of a set of linear matrix inequalities. This formulation enables to use convex optimization methods to design a gain matrix of a state feedback controller in each control step. The task of stabilization is solved in assumed control conditions with respect to symmetric constraints on control inputs. The control performance achieved by robust constrained model-based predictive control is studied via simulations. Obtained results confirm that the robust constrained model-based predictive control ensures the stability demands and the quality requirements represented by chosen quadratic cost function.
\end{abstract}

Keywords: chemical reactor, constrained input, linear matrix inequality, parametric uncertainty, robust MPC

\section{Introduction}

The main task of robust control is to ensure the stability of the feedback control loop and to reach required quality of control performance in the presence of uncertainty in the controlled system. Robust constrained model-based predictive control (MPC) is capable to handle the task of robust control in an effective way taking into account the constraints on control inputs and outputs (Pannocchia et al. 2011).

Continuous stirred tank reactors (CSTRs) represent typical systems with uncertainties because they have parameters varying in certain intervals or constant parameters with not exactly known values. Many authors deal with control of CSTRs. Partial enumeration (PE) method for fast offsetfree robust MPC control of a CSTR is presented in the paper by Pannocchia et al. (2011). The authors show that the strong robust exponential stability of the closed-loop system under PE-based MPC holds for any sufficiently small but otherwise arbitrary perturbation. The disadvantage of the proposed strategy is a suboptimal solution of the optimization problem. Nonlinear adaptive control of a CSTR is studied by Dostál et al. (2011). The control strategy is based on the factorization of the controller into a nonlinear and linear part. The designed controller can be tuned by closed-loop pole assignment. This approach does not handle uncertainties in the controlled process. Favache and Dochain (2010) deal with the design of powershaping control of a non-isothermal CSTR. This control approach for nonlinear systems is based on the physics of the dynamical system. The main limitation of the strategy is the necessity to solve the partial differential equations that represent constraints of an optimization problem. An online iterative algorithm of the robust MPC design for the quasi-linear parameter varying (quasi-LPV) CSTR with bounded disturbance is presented in the paper Ding (2010). The quasi-LPV means that the varying parameters of the linear system are known at the current time, but unknown in the future. The control law is parameterized as a parameter-dependent dynamic output feedback and the closed-loop stability is specified by the notation of quadratic boundedness. Convex optimization problem is solved via linear matrix inequalities (LMIs) techniques. Kvasnica et al. (2010) present application of hybrid MPC applied to a CSTR. A nonlinear system is approximated by piecewise affine (PWA) functions to decrease the model complexity. Accuracy of this strategy was evaluated using simulation of control. The approach does not take into account uncertainties in the controlled system. The on-line constrained robust MPC guaranteeing quadratic stability of linear systems based on the LMIs formulation is presented in Kothare et al. (1996). An advantage of this approach is infinite prediction horizon. On the other hand, only symmetric constraints on control inputs and outputs are considered. Explicit MPC reformulation of previous algorithm is presented in Wan and Kothare (2003). Improved strategy of robust MPC design with reduced range of conservatism and extended feasible set of initial conditions is introduced in Cuzzola et al. (2002). 
This control scenario defines the parameterdepended Lyapunov functions (PDLFs) for each vertex of an uncertain system.

This paper studies robust constrained MPG stabilization of an unstable and uncertain CSTR. Hydrolysis of propylene oxide to propylene glycol runs in the reaction mixture in the reactor (Molnár et al. 2002). Three uncertain technological parameters of the CSTR are considered. Therefore the set of eight different vertex systems was generated. The main task is to ensure stability and required control performance of the whole unstable uncertain system. The considered control algorithm uses infinite prediction horizon. Solution of convex optimization problem is not suboptimal, and conservativeness is reduced using various PDLFs. Symmetric constraints on control inputs are taken into account. The paper is organised as follows. Theoretical backgrounds of the control approach are given at first. Then, the benchmark CSTR and the control conditions are introduced and obtained simulation results are presented. Conclusions are formulated in the last section.

\section{Theoretical}

\section{Uncertain State-Space System}

Suppose that an uncertain linear controlled system is represented by a discrete-time state-space system in the form given by Eq. (1)

$$
\begin{aligned}
& x(k+1)=A x(k)+B u(k), x(0)=0 \\
& y(k)=C x(k) \\
& {[A, B] \in \Omega, \Omega=C o\left(\left[A_{v}, B_{v}\right]\right), v=1, \ldots, N_{v}, k \geq 0}
\end{aligned}
$$

where $k$ represents the discrete time, $\Omega$ is a convex hull of all vertex systems of the system (1) and it is a polytop, $N_{v}$ is the number of vertex systems, $x(k)$ is the $N_{x}$-dimensional vector of states, $u(k)$ is the $N_{u}$-dimensional vector of control inputs, $y(k)$ is the $N_{y}$-dimensional vector of outputs and matrices $A_{v}$, $B_{v}, C_{v}$ have appropriate dimensions. The control input $u(k)$ has to be found so that the quadratic cost function

$$
J=\sum_{k=0}^{N}\left(x(k)^{T} Q x(k)+u(k)^{T} R u(k)\right)
$$

is minimized, where $N$ is the number of control steps and $Q>0, R>0$ are real symmetric weight matrices of system states $x(k)$ and control inputs $u(k)$, respectively. The quality of control depends on the possibility to take into account the symmetric constraints on controlled outputs and control inputs described by Eq. (3)

$$
\begin{gathered}
\|y(k)\|_{2} \leq y_{\max },\|u(k)\|_{2} \leq u_{\max }, \\
\left|u_{j}(k)\right| \leq u_{j, \max }, j=1,2, \ldots, N_{u}
\end{gathered}
$$

where $y_{\max }, u_{\max }$ are the vectors representing the maximal admissible distance from the operating point, $\left|u_{j}(k)\right|$ is the symmetric peak norm, $\|y(k)\|_{2}$ and $\|u(k)\|_{2}$ are the Euclidean norms of system outputs $y(k)$ and inputs $u(k)$, respectively.

\section{LMI-Based Robust Constrained MPC Stabilization}

The main task of robust constrained MPC stabilization is to design a state feedback control law (Kothare et al. 1996, Wan and Kothare 2003, Cuzzola et al. 2002)

$$
u(k)=F_{k} x(k)
$$

where the matrix $F_{k}$ represents the robust controller in the $k$-th control step. To design the gain matrix $F_{k}$, the approach described in Cuzzola et al. (2002) will be applied. For the Lyapunov matrix $P_{k, v}$ of the $v$-th vertex system and for the feedback controller $F_{k}$ following conditions hold

$$
X_{k, v}=\lambda_{k} P_{k, v}^{-1}, Y_{k}=F_{k} W_{k}
$$

where $\lambda_{k}$ is the auxiliary optimization parameter and $X_{k, v}=X_{k, v}{ }^{T}>0, W_{k}>0$ and $Y_{k}$ represent real auxiliary optimization matrices enabling the evaluation of the robust feedback controller matrix $F_{k}$ in the form (Cuzzola et al. 2002)

$$
F_{k}=Y_{k} W_{k}^{-1}
$$

The robust constrained MPC design can be transformed into the solution of a convex optimization problem based on the linear matrix inequalities (LMIs), using substitutions and Schur complement formula (Cuzzola et al. 2002)

$$
\min _{W_{k}, X_{k, v}, Y_{k}} \lambda_{k}
$$

subject to:

$$
\left[\begin{array}{cc}
1 & x_{k}^{T} \\
* & X_{k, v}
\end{array}\right] \succ 0
$$

$\left[\begin{array}{cccc}W_{k}+W_{k}^{T}-X_{k, v} & W_{k}^{T} A_{v}^{T}+Y_{k}^{T} B_{v}^{T} & W_{k}^{T} \sqrt{Q} & Y_{k}^{T} \sqrt{R} \\ * & X_{k, v} & 0 & 0 \\ * & * & \lambda_{k} I & 0 \\ * & * & * & \lambda_{k} I\end{array}\right] \succ 0$, $v=1, \ldots, N_{v}$

where the symbol * denotes the symmetric structure of the matrix and $I$ represents an identity matrix of appropriate dimensions. The LMIs represent powerful technique to obtain the control problem in more tractable form of convex optimization problem that is solved in each control step in effective way. 
The symmetric Euclidean norm constraints on control inputs $u(k)$ in the form of Eq. (3) can be added to the convex optimization problem described by Eqs. (7)-(9) in the form of the following LMI

$$
\left[\begin{array}{cc}
u_{\max }^{2} I & Y_{k} \\
* & W_{k}+W_{k}^{T}-X_{k, v}
\end{array}\right] \succ 0
$$

The symmetric peak norm constraints on control inputs $u(k)$ in the form of Eq. (3), can be added to the optimization problem Eqs. (7)-(9) in the following LMI form

$$
\left[\begin{array}{cc}
U_{k} & Y_{k} \\
* & W_{k}+W_{k}^{T}-X_{k, v}
\end{array}\right] \succ 0
$$

where for each diagonal element of the matrix $U_{k}$ hold the inequality (Cuzzola et al. 2002)

$$
U_{j, j}(k) \leq u_{j, \max }^{2}, j=1, \ldots, N_{u}
$$

Similarly, the symmetric Euclidean norm constraints on controlled outputs $y(k)$ in the form of Eq. (3), can be added into optimization problem in the following LMI form

$$
\begin{aligned}
& {\left[\begin{array}{cc}
W_{k}+W_{k}^{T}-X_{k, v} & \left(A_{v} W_{k}+B_{v} Y_{k}\right)^{T} C^{T} \\
* & y_{\max }^{2} I
\end{array}\right] \geq 0,} \\
& v=1, \ldots, N_{v}
\end{aligned}
$$

The convex optimization problem is solved in each control step $k$ of the discrete-time domain, therefore it is necessary to find proper value of sampling time $t_{\mathrm{S}}$. Then the algorithm of robust constrained MPC can be formulated as follows (Cuzzola et al. 2002):

1. Set initial value of the control step parameter $k=0$.

2. Set required number of control steps $N$, initial conditions of system states $x(0)$, values of the symmetric constraints on control inputs $u_{\max }$ and outputs $y_{\max }$.

3. Set control step parameter $k=k+1$.

4. Measure or estimate the values of system states $x(k)$.

5. Solve the convex optimization problem described by Eqs. (7)-(13) to evaluate the matrices $W_{k}, X_{k}$ and $Y_{k}$.

6. Design the gain matrix $F_{k}$ of the feedback controller using Eq. (6).

7. Calculate the control input $u(k)$ using the control law Eq. (4).

8. If the parameter $k<N$ then go to the Step 3 else Stop.

\section{Experimental}

\section{Controlled Process Description}

Continuous-time stirred reactor (CSTR) has been adopted from (Molnár et al. 2002) and represents the controlled process. The propylene glycol $\left(\mathrm{C}_{3} \mathrm{H}_{8} \mathrm{O}_{2}\right)$ is produced in the reactor by hydrolysis of propylene oxide $\left(\mathrm{C}_{3} \mathrm{H}_{6} \mathrm{O}\right)$. The exothermic firstorder chemical reaction with respect to propylene oxide as a key component is

$$
\mathrm{C}_{3} \mathrm{H}_{6} \mathrm{O}+\mathrm{H}_{2} \mathrm{O} \stackrel{\mathrm{CH}_{3} \mathrm{OH}}{\longrightarrow} \mathrm{C}_{3} \mathrm{H}_{8} \mathrm{O}_{2}, \Delta_{r} H<0
$$

The methanol $\left(\mathrm{CH}_{3} \mathrm{OH}\right)$ is added into reaction mixture to improve solubility of propylene oxide in water $\left(\mathrm{H}_{2} \mathrm{O}\right)$. The excess of water ensures higher selectivity of the main product and prevents ineligible side reactions (Molnár et al. 2002). Hereafter the subscripts PO and PG denote source compound propylene oxide and main product propylene glycol, respectively. The parameters of the CSTR are summarized in the Table 1 , where $V, V_{\mathrm{C}}$ are volumes of the reactor vessel and cooling jacket, respectively. Parameters $\rho, \rho_{\mathrm{C}}$ denote densities, $c_{p}, c_{p, \mathrm{C}}$ are thermal capacities, $q, q_{\mathrm{C}}$ are volumetric flow rates and $T_{0}, T_{\mathrm{C}, 0}$ are inlet temperatures of reaction mixture and cooling medium, respectively. Coefficient $A_{\mathrm{h}}$ is the heat transfer area of the reactor vessel and the constant $g$ represents the ratio of activation energy and universal molar gas constant.

Tab. 1. Parameters of the CSTR.

\begin{tabular}{lcc}
\hline Variable & Value & Unit \\
\hline$V$ & 2.4 & $\mathrm{~m}^{3}$ \\
$V_{\mathrm{C}}$ & 2.0 & $\mathrm{~m}^{3}$ \\
$\rho$ & 947.19 & $\mathrm{~kg} \mathrm{~m}^{-3}$ \\
$\rho_{\mathrm{C}}$ & 998.00 & $\mathrm{~kg} \mathrm{~m}^{-3}$ \\
$c_{P}$ & 3.719 & $\mathrm{~kJ} \mathrm{~kg}^{-1} \mathrm{~K}^{-1}$ \\
$c_{P, \mathrm{C}}$ & 4.182 & $\mathrm{~kJ} \mathrm{~kg}^{-1} \mathrm{~K}^{-1}$ \\
$q$ & 0.0720 & $\mathrm{~m}^{3} \mathrm{~min}^{-1}$ \\
$q_{\mathrm{C}}$ & 0.6307 & $\mathrm{~m}^{3} \mathrm{~min}^{-1}$ \\
$c_{\mathrm{PO}}$ & 0.0824 & $\mathrm{kmol} \mathrm{m}^{-3}$ \\
$c_{\mathrm{PG}}$ & 0.0000 & $\mathrm{kmol} \mathrm{m}^{-3}$ \\
$T_{0}$ & 299.1 & $\mathrm{~K}$ \\
$T_{\mathrm{C}, 0}$ & 288.6 & $\mathrm{~K}$ \\
$A_{\mathrm{h}}$ & 8.695 & $\mathrm{~m}$ \\
$g$ & 10183.0 & $\mathrm{~K}$ \\
\hline
\end{tabular}

The admissible boundary values of uncertain parameters are in the Table 2 , where $\Delta_{\mathrm{r}} H$ is the reaction enthalpy of the exothermic reaction described by Eq. (14), $k_{0}$ denotes the pre-exponential factor of Arrhenius equation, see e.g. Bakošová et al. (2009), and $U$ represents the heat transfer coefficient. 
Tab. 2. Uncertain Parameters of the CSTR.

\begin{tabular}{lccc}
\hline Variable & Minimal Value & Maximal Value & Unit \\
\hline$\Delta_{\mathrm{r}} H$ & $-5.64 \times 10^{6}$ & $-5.28 \times 10^{6}$ & $\mathrm{~kJ} \mathrm{kmol}^{-3}$ \\
$k_{0}$ & $2.4067 \times 10^{11}$ & $3.2467 \times 10^{11}$ & $\mathrm{~min}^{-1}$ \\
$U$ & 13.11 & 14.49 & $\mathrm{~kJ} \mathrm{~min}^{-1} \mathrm{~m}^{-2} \mathrm{~K}^{-1}$ \\
\hline
\end{tabular}

The continuous-time model of the CSTR is composed of four differential equations based on the material and enthalpy balances, see Molnár et al. (2002), Bakošová and Oravec (2012), Bakošová et al. (2009).

Nominal system is the model of the CSTR obtained using mean values of interval parametric uncertainties (Table 2). Vertex systems are models generated for all combinations of all boundary values of uncertain parameters. For the controlled system with three interval parametric uncertainties $N^{v}=2^{3}=8$ vertex systems were generated. The set $\Omega$ given by Eq. (1) is defined as a convex hull of vertex systems. The convex hull includes all possible uncertain systems. Therefore the set of investigated systems is reduced into the eight vertex systems and the set is extended by the nominal system of the CSTR as the reference system. The final set of nine systems was transformed from the continuous-time into the discrete-time domain using sampling time $t_{\mathrm{S}}=0.5 \mathrm{~min}$.

\section{Results and Discussion}

The steady-state analysis of the CSTR was done at first (Bakošová and Oravec, 2012). The results obtained for the nominal system are displayed in the Fig. 1, where the curve $Q_{\mathrm{GEN}}$ (solid) represents heat generated by the exothermic reaction given in Eq. (14) and the line $Q_{\text {out }}$ (dashed) denotes the heat withdrawn from the reaction vessel by the product stream and cooling jacket.

The steady states of the CSTR are represented by the intersections of the curves. The steady state is stable if and only if the slope of the $Q_{\text {out }}$ line is higher than the slope of the $Q_{\mathrm{GEN}}$ curve. As can be seen in Fig. 1, the reactor can operate in three steady states. The stability condition is satisfied in the steady-states at the temperatures $T_{1}^{\mathrm{S}}=296.7 \mathrm{~K}$ and $T_{3}{ }^{\mathrm{S}}=377.5 \mathrm{~K}$ and is not satisfied at the temperature $T_{2}{ }^{\mathrm{S}}=343.1 \mathrm{~K}$. The steady-state analysis of all vertex systems gives similar results. Control of reactors around theirs unstable steady-states is often interesting because of economic performance or safety problems. Hence the aim of robust control of the described CSTR is to stabilize it in the surroundings of the unstable steady-state at the temperature 343.1 K so that the cost function given by Eq. (2) is minimized, where $Q=\operatorname{diag}\left([100 ; 100 ; 0.01 ; 0.01]^{\mathrm{T}}\right)$

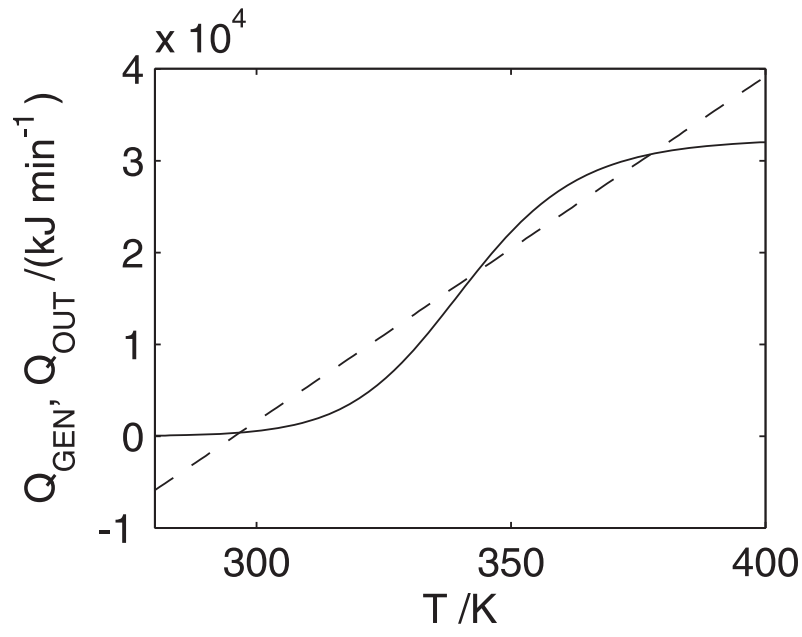

Fig. 1. Steady-state analysis of CSTR with nominal values of uncertain parameters $Q_{\text {GEN }}$ (solid), $Q_{\text {OUT }}$ (dashed).

and $R=\operatorname{diag}\left([100 ; 100]^{\mathrm{T}}\right)$. The controlled outputs are temperatures in reaction vessel $T$ and cooling jacket $T_{\mathrm{C}}$. Assumed control inputs are volumetric flow rates of reaction mixture $q$ and cooling medium $q_{\mathrm{C}}$. Thus we have multiple-input and multipleoutput (MIMO) system. The constraints on control inputs were considered to prevent calculating negative values of flow rates, therefore $u_{\max }=[0.0720$; $0.6307]^{\mathrm{T}}$ in Eq. (3). Initial conditions of the state variables of the reactor in simulations were $c_{\mathrm{PO}}(0)=0.0291 \mathrm{kmol} \mathrm{m}^{-3}, c_{\mathrm{PG}}(0)=0.0533 \mathrm{kmol} \mathrm{m}^{-3}$, $T(0)=349.1 \mathrm{~K}, T_{\mathrm{C}}(0)=296.6 \mathrm{~K}$.

The simulations were realized in the MATLABSimulink environment by $3.2 \mathrm{GHz}$ CPU and 4 GB RAM. The problem of convex optimization was solved using YALMIP toolbox (Löfberg 2004) with solver SeDuMi (Pólik 2010). The control performance ensured by the designed robust constrained MPC approach was studied by simulation of control of 9 linear models of the reactor - nominal and vertex systems - in the form given by Eq. (1).

Obtained trajectories of controlled outputs are shown in Fig. 2. Fig. 2a) presents the control performance of the temperature of reaction mixture $T$ and Fig. 2b) displays the temperature of cooling medium $T_{C}$ of the nominal system (dashed) and all vertex systems (solid). The operating point represents the unstable steady-state and is denoted by 


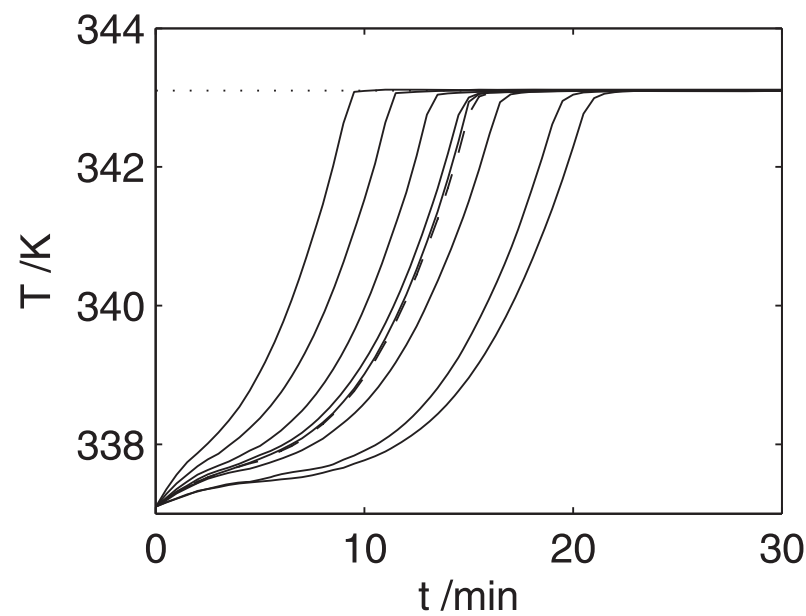

a)

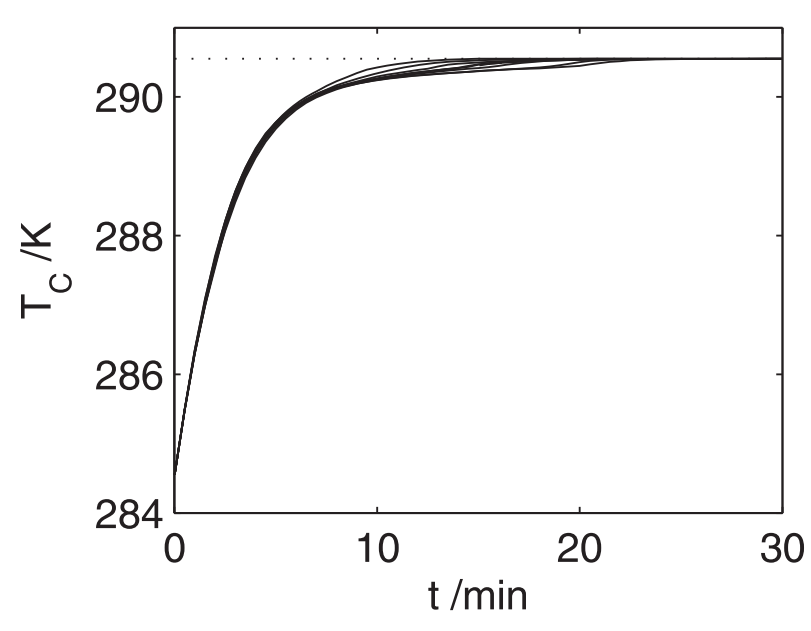

b)

Fig. 2. Control performance ensured by robust constrained MPC: a) temperature of reaction mixture, b) temperature of cooling medium - nominal system (dashed), vertex systems (solid) and reference (dotted).

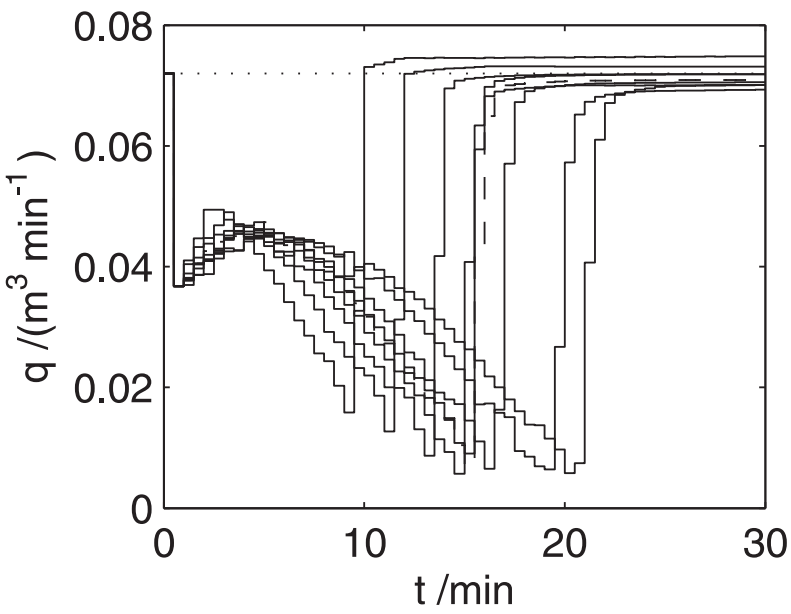

a)

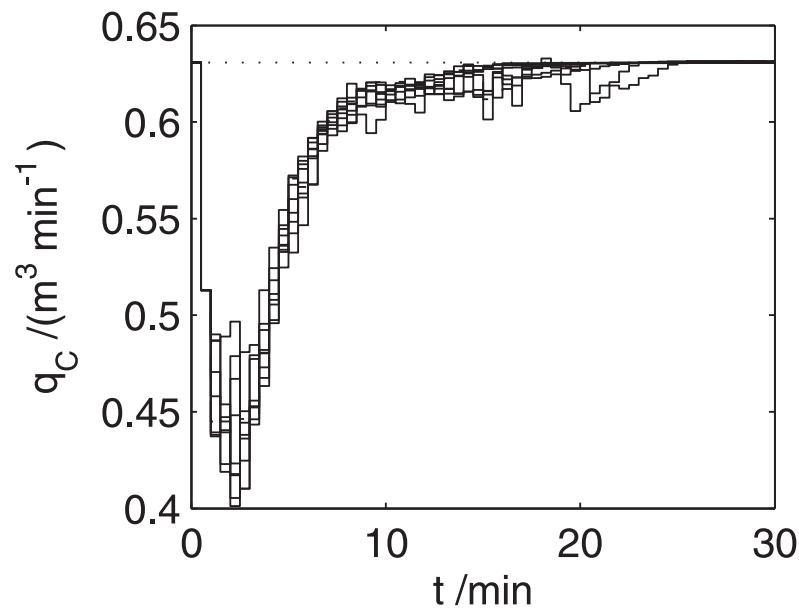

b)

Fig. 3. Control inputs generated by robust constrained MPC: a) flow rate of reaction mixture b) flow rate of cooling medium - nominal system (dashed) and vertex systems (solid)

the dotted line. As can be seen in Fig. 2, controlled outputs converge to the unstable steady-state values $T_{2}^{\mathrm{S}}=343.1 \mathrm{~K}, T_{\mathrm{C}, 2}{ }^{\mathrm{S}}=290.6 \mathrm{~K}$. Fig. 3 shows the control inputs, where Fig. 3a) presents the volumetric flow rate of reaction mixture $q$ and Fig. 3b) shows the volumetric flow rate of the cooling medium $q_{\mathrm{C}}$ in the nominal system (dashed) and all considered vertex systems (solid). It is proved in Fig. 3, the generated values of control inputs respect given symmetric constraints. The nominal value of the cost function given by Eq. (2) is $J_{\mathrm{NOM}}=10.6401$. The maximal worst-case value of the cost function obtained for one of vertex systems is $J_{\mathrm{WC}}=14.1692$. The values of cost functions were calculated for simulation time $100 \mathrm{~min}$ after which all variables were settled. Although the worst-case scenario evoked increasing of the cost function value about $33 \%$ in comparison with the nominal system, the robust constrained MPC stabilized the reactor with uncertain parameters in all investigated situations and kept control inputs within given constraints.

\section{Conclusions}

The paper presents an on-line concept of the robust constrained MPC of the CSTR. The algorithm is based on the LMI formulation that enables to solve the convex optimization problem in each control step in an effective way. Operating point of the CSTR was its unstable steady-state. Control performance of the CSTR assured using the robust MPG was tested via simulation of control of the nominal and eight 
vertex systems. The vertex systems were obtained for all combinations of boundary values of all considered interval parametric uncertainties. Obtained simulation results confirm that the designed robust constrained MPG is capable to stabilize the CSTR with uncertainties into the unstable operating point taking into account the symmetric constraints on control inputs.

\section{Acknowledgement}

The authors gratefully acknowledge the contribution of the Scientific Grant Agency of the Slovak Republic under the grants 1/0973/12 and 1/0095/11.

\section{References}

Bakošová M, Oravec J (2012) Proc. of the $39^{\text {th }}$ Int. Conf. of SSChE: 5-16.
Bakošová M, Puna D, Vasičkaninová A, Karšaiová M (2010) Selected Topics in Modelling and Control 6: $19-25$.

Cuzzola FA, Geromel JG, Morari M (2002) Automatica 38: 1181-1189.

Ding B (2010) Automatica 46: 1517-1523.

Dostál P, Bakošová M, Vojtěšek J, Bobál V (2011) Chemical Papers 5: 636-643.

Favache A, Dochain D (2010) Automatica 46: 1877-1883.

Kothare MV, Balakrishnan V, Morari M (1996) Automatica 32: 1361-1379.

Kvasnica M, Herceg M, Čirka L', Fikar M (2010) Chemical Papers 3: 301-309.

Löfberg J (2004) Proc. of the CACSD Conference: 284-289.

Molnár A, Markoš J, Jelemenský L' (2002) Chemical Papers 56: 357-361.

Pannocchia G, Wright SJ, Rawlings JB (2011) Journal of Process Control 21: 1458-1466.

Pólik I (2010) http://sedumi.ie.lehigh.edu/.

Wan Z, Kothare MV (2003) Automatica 39: 837-846. 